\title{
Magnetic antidot to dot crossover in Co and Py nanopatterned thin films
}

\author{
C. Castán-Guerrero, ${ }^{1,2,{ }^{*}}$ J. Herrero-Albillos, ${ }^{1,3,4}$ J. Bartolomé, ${ }^{1,2}$ F. Bartolomé, ${ }^{1,2}$ L. A. Rodríguez, ${ }^{5,2,6}$ \\ C. Magén, ${ }^{5,2,3}$ F. Kronast, ${ }^{7}$ P. Gawronski, ${ }^{8}$ O. Chubykalo-Fesenko, ${ }^{9}$ K. J. Merazzo, ${ }^{9}$ P. Vavassori, ${ }^{10}$ \\ P. Strichovanec, ${ }^{5,2}$ J. Sesé, ${ }^{5,2}$ and L. M. García ${ }^{1,2}$ \\ ${ }^{1}$ Instituto de Ciencia de Materiales de Aragón (ICMA), CSIC - Universidad de Zaragoza, E-50009, Zaragoza, Spain \\ ${ }^{2}$ Departamento de Física de la Materia Condensada, Universidad de Zaragoza, E-50009, Zaragoza, Spain \\ ${ }^{3}$ Fundación ARAID, E-50018, Zaragoza, Spain \\ ${ }^{4}$ Centro Universitario de la Defensa, E-50090, Zaragoza, Spain \\ ${ }^{5}$ Instituto de Nanociencia de Aragón (INA) and Laboratorio de Microscopías Avanzadas (LMA), \\ Universidad de Zaragoza, E-50018, Zaragoza, Spain \\ ${ }^{6}$ CEMES-CNRS, 29 rue Jeanne Marvig, B.P. 94347, F-31055 Toulouse Cedex, France \\ ${ }^{7}$ Helmholtz-Zentrum Berlin für Materialien und Energie GmbH, 12489, Berlin, Germany \\ ${ }^{8}$ Faculty of Physics and Computer Science, AGH University of Science and Technology, 30-059, Cracow, Poland \\ ${ }^{9}$ Instituto de Ciencia de Materiales de Madrid, Consejo Superior de Investigaciones Científicas, E-28049, Madrid, Spain \\ ${ }^{10}$ CIC nanoGUNE Consolider, E-20018, San Sebastian, Spain and IKERBASQUE, Basque Foundation for Science, E-48011, Bilbao, Spain
}

(Received 25 June 2013; revised manuscript received 6 February 2014; published 4 April 2014)

\begin{abstract}
The crossover from antidot to dot magnetic behavior on arrays patterned in a ferromagnetic thin film has been achieved by modifying only the geometry. A series of antidot arrays has been fabricated on cobalt with fixed diameter $d$ and by reducing the period of the array $p$ from $p \gg d$ to $p<d$. A dramatic change in the coercivity dependence with $p$, correlated with a significant modification in the magnetic domain structure observed by $\mathrm{x}$-ray photoemission electron microscopy, evidences the crossover. An intermediate regime has been found between the superdomain structure present in antidot arrays and the array of astroid-state noncorrelated dots. The study has been reproduced for a different ferromagnetic material, permalloy, and supported by micromagnetic simulations.
\end{abstract}

DOI: 10.1103/PhysRevB.89.144405 PACS number(s): 75.75.-c, 68.37.Xy, 75.60.-d, 81.16.Nd

\section{INTRODUCTION}

The control of the magnetic properties of ferromagnetic thin films is an important topic for both the fundamental understanding of low-dimensional magnetism and a broad range of applications, such as magnetic recording media [1], sensors [2], and magnonic devices [3,4]. A strong dependence of the magnetization reversal mechanisms on the microstructure and the presence of defects makes magnetic properties hardly controllable. Nevertheless, the astonishing development of nanofabrication techniques [5-8] in recent years has opened the door to a new strategy, the patterning of nanostructures, that allows the modification of the local magnetization distribution in a controlled way. Peculiar magnetic domain structures appear on thin film surfaces, such as quasidomains [9], or in nanostructured thin films, such as hyperdomains [10] or superdomains [11]. The relevant characteristic length scale for magnetic phenomena in thin films is the exchange length [9] $\Delta_{d}=\sqrt{2 A / \mu_{0} M_{S}^{2}}$ ( $A$ : exchange stiffness constant; $M_{S}$ : saturation magnetization). In the present work polycrystalline cobalt and permalloy thin films have been studied, both with $\Delta_{d} \approx 5 \mathrm{~nm}$. Two patterning strategies allowing to reach this lengthscale are the fabrication of dot arrays, and their negative pattern, arrays of nonmagnetic inclusions or holes, the so-called antidot arrays.

On the one hand, antidots are known to change the magnetization switching mechanisms [12], acting as pinning centers for domain walls (DWs) [13] and enabling novel magnetic domain configurations connected to the array geometry [14-18]

\footnotetext{
*ccastan@unizar.es
}

such as superdomains [11], that are not observed in an unpatterned film. The antidot geometry can also be used to tailor the coercivity $H_{C}$ : many previous studies report an empirical law according to which $H_{C}$ increases linearly with the inverse of the antidot edge-to-edge separation $\lambda=p-d$, where $d$ is the antidot diameter and $p$ the array period, for both $\lambda \gg \Delta_{d}[19,20]$ and $\lambda \gtrsim \Delta_{d}[21-23]$.

On the other hand, in dot arrays the single domain state $[24,25]$ is favored for dots small compared to $\Delta_{d}$, with in-plane or out-of-plane magnetization when the largest dimension is the lateral size or thickness, respectively [26,27]. Moreover, vortex and multidomain states are the stable configurations for large dots. Nonhomogeneous structures such as flower, leaf, $C$ states, or $S$ states $[25,27,28]$ can be also stabilized in small dots with different shapes. This shape effect can drastically change the magnetic properties [28] making it difficult to establish a general dependence on dot size.

All that said, in the present work the magnetic crossover from antidot to dot arrays when $\lambda$ is positive and negative, respectively, has been studied. The focus has been placed in the intermediate state, taking place when $\lambda \lesssim \Delta_{d}$. This structural and magnetic crossover has been driven, with the material parameters remaining unchanged, by just varying the geometry. In Sec. II, the patterning of antidot arrays on thin films is described. Focused ion beam (FIB) etching [29] has provided access to patterning dimensions of the order of the relevant length scale $\Delta_{d}$. Three series of antidot arrays with different materials (cobalt and permalloy) and geometrical parameters have been fabricated. $d \approx 60 \mathrm{~nm}$ in all the series. In Series 1, fabricated on cobalt, $105<p<$ $500 \mathrm{~nm}$, while in Series 2-Co and 2-Py, fabricated on cobalt and permalloy, respectively, $150<p<2000 \mathrm{~nm}$. The structural 

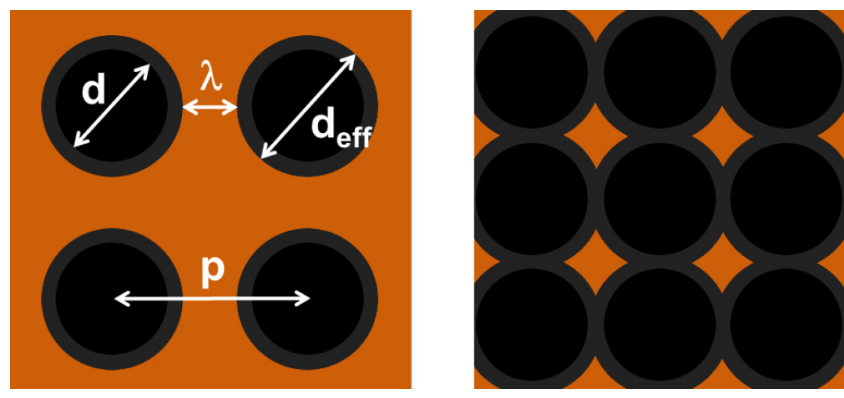

FIG. 1. (Color online) Left: Definition of the geometrical parameters of the arrays: $p, d, d_{\text {eff }}$, and $\lambda$. Orange color represents the magnetic material, while black areas are nonmagnetic holes with diameter $d$, and grey areas are the holes with effective diameter $d_{\text {eff }}$. Right: Scheme of the astroid-shaped dots formed by the intersection of the holes in the dot $(D)$ regime, where $p<d_{\text {eff }}$.

and chemical characterization of the arrays has been conducted by transmission electron microscopy, as presented in Sec. IV. The magnetic characterization by means of the magnetooptical Kerr effect (MOKE) and x-rays photoelectron microscopy (XPEEM) is shown in Sec. V. An effective antidot diameter, $d_{\text {eff }}$, larger than the morphological hole diameter measured in scanning electron microscopy (SEM) images, is obtained from MOKE measurements. It is consistent with the FIB damage around the holes observed in Sec. IV. The broadening of the effective diameter gives rise to the case with $p<d_{\mathrm{eff}}$, that is, $\lambda<0$, where antidots intersect with each other, corresponding to the dot regime (see Fig. 1). The dependence of $H_{C}$ on $p$, obtained from MOKE hysteresis loops, has been correlated with XPEEM images of the magnetic domains with nanometric resolution, revealing that the crossover from antidot to dot regime takes place continuously through an intermediate state, which is analyzed in detail. In Sec. VI, micromagnetic simulations have been performed supporting the obtained results.

\section{SAMPLE FABRICATION}

Three series of square arrays of circular antidots have been fabricated with different geometrical parameters on two magnetic materials: cobalt and permalloy. Polycrystalline films with $10-\mathrm{nm}$ thickness of cobalt $(\mathrm{Co})$ and permalloy (Py, i.e., $\mathrm{Ni}_{80} \mathrm{Fe}_{20}$ ) were deposited on silicon substrates by dc-magnetron sputtering. A 2-nm capping layer of aluminum was deposited on top of the films to prevent their oxidation. Co films were deposited with an AJA International ATC ORION 5 system with base pressure $6 \times 10^{-8}$ torr. For the Py film, a homemade magnetron sputtering system was used, with base pressure of $2.25 \times 10^{-6}$ torr.

Antidots have been etched by means of $\mathrm{a} \mathrm{Ga}^{+}$focused ion beam (FIB), with a FEI Helios 650 Dual Beam system. The equipment also has a focused electron beam, $52^{\circ}$ tilted with respect to the ion beam, that allows to obtain SEM images during fabrication. Each hole is etched with a single spot of the ion beam, and antidot arrays are fabricated by deflecting the beam from point to point. The hole diameter depends on the ion beam current and acceleration voltage that have been fixed to $28 \mathrm{pA}$ and $30 \mathrm{kV}$, respectively, and the dwell time of
TABLE I. Antidot diameter for all the series of arrays.

\begin{tabular}{lccclc}
\hline & Material & Dwell time & $d(\mathrm{~nm})$ & $d_{\text {ext }}(\mathrm{nm})$ & $d_{\text {eff }}(\mathrm{nm})$ \\
\hline Series 1 & Co & $10 \mathrm{~ms}$ & $61 \pm 2$ & $107 \pm 8$ & $114 \pm 4$ \\
Series 2 & a: Co & $25 \mathrm{~ms}$ & $60 \pm 3$ & $130 \pm 10$ & $169 \pm 4$ \\
& b: Py & $25 \mathrm{~ms}$ & $62 \pm 7$ & $134 \pm 11$ & $166 \pm 4$ \\
\hline \hline
\end{tabular}

the beam at each position. The last of these has been set to $10 \mathrm{~ms}$ in Series 1, which was fabricated in cobalt, and to $25 \mathrm{~ms}$ in Series 2-Co and 2-Py, patterned in cobalt and permalloy, respectively. SEM images of some of the arrays in Series 1 are shown in Fig. 2. The black circles in the SEM images, with a diameter $d=61 \pm 2 \mathrm{~nm}$, correspond to the areas in which the ion beam has completely perforated the cobalt film. In the inset of Fig 2(b), a tilted SEM image gives a clearer view of the three-dimensional shape of the holes, and the parameters $p, d$, and $d_{\text {ext }}$ are defined. The dark grey tone in a ring surrounding the holes, with diameter $d_{\text {ext }}=107 \pm 8 \mathrm{~nm}$, is due to a gradient in cobalt thickness caused by the Gaussian-like section of the ion beam. The antidot diameter obtained in Series 2-Co and Py is $d=60 \pm 3$ and $62 \pm 7 \mathrm{~nm}$, respectively, which is close to that in Series 1. However, as a result of a larger dwell time, $d_{\mathrm{ext}}=130 \pm 10$ and $134 \pm 11 \mathrm{~nm}$ for Series 2-Co and Py, respectively, is larger than the corresponding one in Series 1.

In Table I the dwell time used in each series and the obtained antidot diameter are summarized. While $d$ is fixed within the different arrays of each series, $p$ is changed from array to array. The complete set of $p$ values of each series can be found in Table II. Each array in Series 1 covers a square area of $20 \times 20 \mu \mathrm{m}^{2}$, surrounded by a $5-\mu \mathrm{m}$ wide trench in which the magnetic material has been removed to isolate the array from the unpatterned film. In Series 2, the arrays cover a square area of $10 \times 10 \mu \mathrm{m}^{2}$, with a $1-\mu \mathrm{m}$ wide trench.

\section{EXPERIMENTAL DETAILS}

High resolution transmission electron microscopy (HRTEM) experiments were carried out in a FEI Titan Cube 60-300 operated at $300 \mathrm{kV}$. This instrument is equipped with an image aberration corrector to provide subangstrom spatial resolution in TEM. Spectrum imaging experiments combining scanning transmission electron microscopy (STEM) and energy dispersive $\mathrm{x}$-ray spectroscopy (EDS) at $300 \mathrm{kV}$ were performed in a FEI Titan 60-300 equipped with a probe aberration corrector, a high-brightness field emission gun (X-FEG) and an energy dispersive $\mathrm{X}$-ray microanalysis (EDXMA) system. The probe size used for the chemical maps was around $1 \AA$. The TEM transverse cross-section lamellae studied in this work have been prepared by FIB in a Helios Nanolab system.

Two different techniques have been used for the room temperature magnetic characterization. First, local hysteresis loops of all the arrays have been measured using a wide field Kerr Effect EVICO@ microscope, with polarization analysis control operated in longitudinal configuration [30] under in-plane applied magnetic field. The light source is a halogen lamp. The image of the sample surface is formed through a $100 \times$ objective and is collected by a high sensitivity 
TABLE II. Period of the arrays in Series 1 and Series 2.

\begin{tabular}{|c|c|c|c|c|c|c|c|c|c|c|c|c|}
\hline Series 1: $p(\mathrm{~nm})$ & 105 & 120 & 130 & 140 & 150 & 175 & 200 & 250 & 300 & 350 & 400 & 500 \\
\hline Series 2: $p(\mathrm{~nm})$ & 150 & 175 & 200 & 300 & 400 & 500 & 750 & 1000 & 2000 & & & \\
\hline
\end{tabular}

Hamamtsu HPF-ORCA-03G charge-coupled device (CCD) camera. In principle, images of the magnetic domains could be obtained with this technique, but the resolution, limited by the Rayleigh criterion, is not enough for that purpose. However, hysteresis loops of each array can be obtained by selecting the corresponding region of interest in the image, i.e., a subset of the pixels of the $\mathrm{CCD}$, and using it as a conventional photodiode. The magnetic sensitivity direction (MSD) in this measurements is given by the in-plane projection of the exchanged wave vector. Magnetic field was applied in-plane up to $\pm 2500 \mathrm{Oe}$, along the direction of the antidot rows, which is parallel to the MSD.

To obtain images with enough spatial resolution and magnetic sensitivity, the XPEEM technique has been used in the UE49-PGM-1-SPEEM beamline of the BESSY II synchrotron (HZB, Berlin). To obtain what we refer to as an X-rays magnetic circular dichroism (XMCD) image [31], circularly polarized $\mathrm{x}$ rays, whose energy is tuned to the $\mathrm{Co}_{3}$ absorption edge, incide at a low angle $\left(16^{\circ}\right)$ on the sample, while images are taken with the photoemission electron microscope both for $x$ rays' left and right circular polarizations. As in regular $\mathrm{XMCD}$, the difference of these two images normalized by their sum provides an image with magnetic contrast proportional to the projection of the magnetization along the direction of the incident beam wave vector, that is, the magnetic sensitivity direction (MSD). Due to the low angle of incidence, the equipment is mostly sensitive to the in-plane component of the magnetization. The spatial resolution in the image is around $30 \mathrm{~nm}$ after a careful focusing of the microscope.

\section{STRUCTURAL AND CHEMICAL CHARACTERIZATION}

Two selected arrays from Series 2-Co have been chosen for the TEM analysis, with $p=500 \mathrm{~nm}$ and $p=175 \mathrm{~nm}$. A lamella has been carefully fabricated in each array, to obtain thin slices of the Co film cutting through a row of nearest neighboring antidots. HRTEM images have been collected to analyze the morphology, microstructure, and crystalline quality of cobalt around the antidots in the array.

Figures 3(a) and 3(b) depict low magnification bright field TEM images of the arrays of $p=500$ and $175 \mathrm{~nm}$, respectively. The samples were protected with electron-beam deposited Pt before FIB thinning, and therefore the antidots have been filled with Pt. A high resolution TEM image [Fig. 3(c)] in the middle point between two holes of the $p=500 \mathrm{~nm}$ array reveals the structure of the sample when it is not altered by the ion beam. Over the crystalline Si substrate, a layer of around $30 \mathrm{~nm}$ of amorphous $\mathrm{Si}$ is present. On top of that, we find the 10-nm thick polycrystalline cobalt film, with grains of few nanometers size and hexagonal close-packed crystal structure, capped with $2 \mathrm{~nm}$ of aluminum.

In both cases, the lamellae have not been perfectly cut through the center of the antidots, as some cobalt signal is superimposed to the contrast coming from the Pt filling the antidot. The areas around the antidots appear altered by the FIB etching. In the sample with $p=500 \mathrm{~nm}$, approximately $30 \mathrm{~nm}$ of cobalt around the antidot have been modified. This is hinted by a lighter tone (lower density in bright field TEM) in the nearby region of the film, which indicates chemical diffusion or alloying between the Si substrate and the cobalt film. A second effect caused by the FIB etching is revealed by the halo in the $\mathrm{Si}$ substrate, about $100 \mathrm{~nm}$ below the antidot, which corresponds to amorphized silicon. Local silicon amorphization is most probably a side-effect of local heating and/or Ga implantation caused by high-energy ion etching.

In the case of $p=175 \mathrm{~nm}$, most of the region between the antidots appears drastically modified. Cobalt film thickness at the middle point between antidots is close to $10 \mathrm{~nm}$, but the FIB etching process has thinned and bent down the cobalt film nearby the antidots. The amorphous Si halo below the antidots is also present in this sample.

The effect of FIB etching in the chemical composition of Co antidot structure has been analyzed by STEM-EDX spectrum

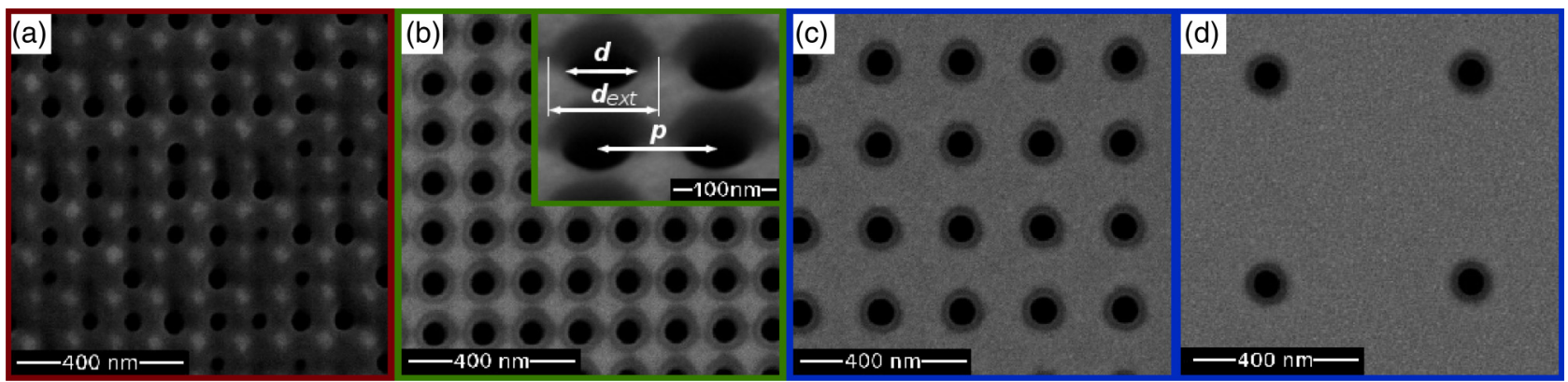

FIG. 2. (Color online) SEM images of some of the arrays in Series 1 , with $p=$ (a) 105, (b) 120, (c) 200, and (d) $500 \mathrm{~nm}$. The inset in (b) shows a zoomed-out and $52^{\circ}$ tilted SEM image of the array with $p=120 \mathrm{~nm}$, in which the parameters $d, d_{\mathrm{ext}}$, and $p$ are indicated. In the arrays with small $p$, silicon etched from the substrate redeposit over the neighboring holes, causing the antidot diameter to seem smaller, as is the case in (a), where some of the antidots have been almost filled up with Si redeposit. 

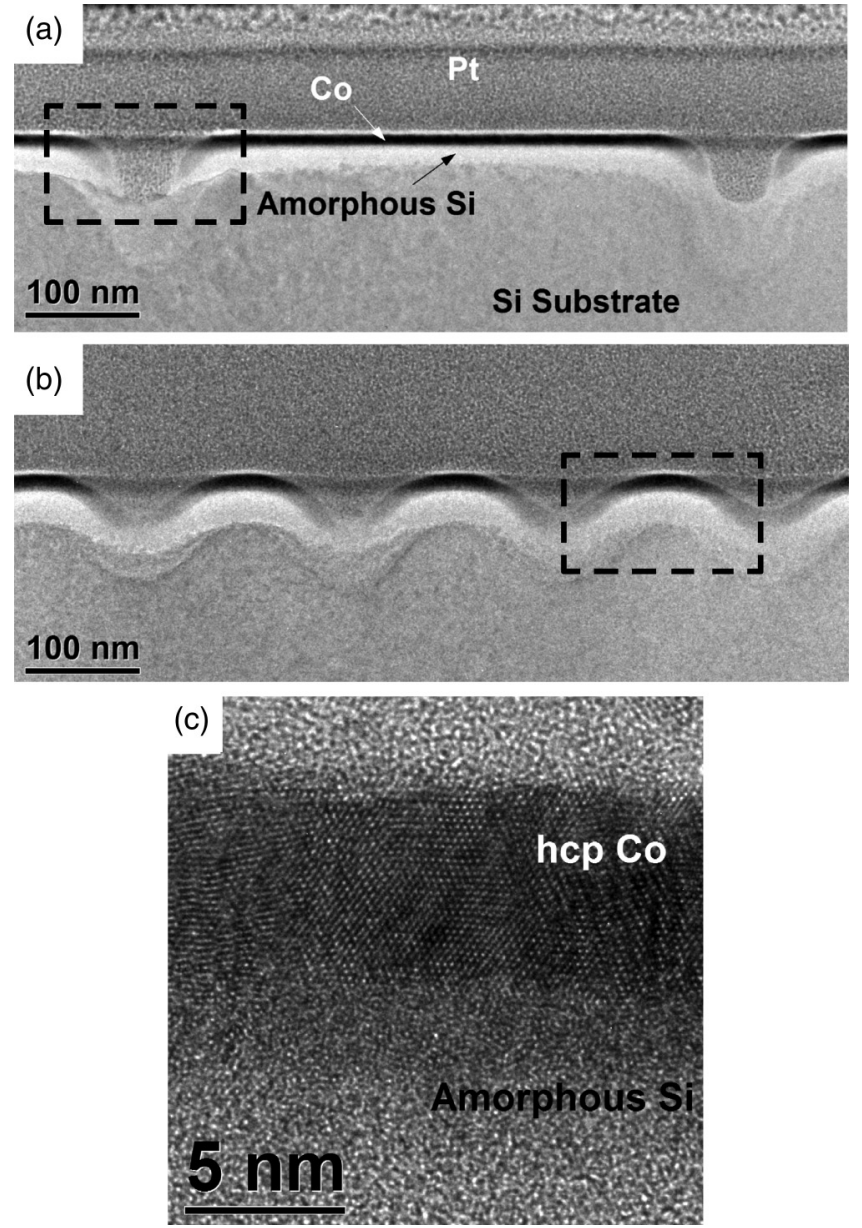

FIG. 3. Low magnification bright field TEM images of arrays in Series 2-Co with (a) $p=500 \mathrm{~nm}$ and (b) $p=175 \mathrm{~nm}$. Dashed squares in $(a, b)$ hallmark the areas in which the chemical mapping at Figs. 4 and 5 has been performed. (c) High resolution TEM image of $p=$ $500 \mathrm{~nm}$.

imaging. The results are summarized in Figs. 4 and 5 for the sample with $p=500$ and $175 \mathrm{~nm}$, respectively. For $p=$ $500 \mathrm{~nm}$ the cobalt film remains unaffected in the areas distant from the antidots. However, $20-30 \mathrm{~nm}$ around the antidot the

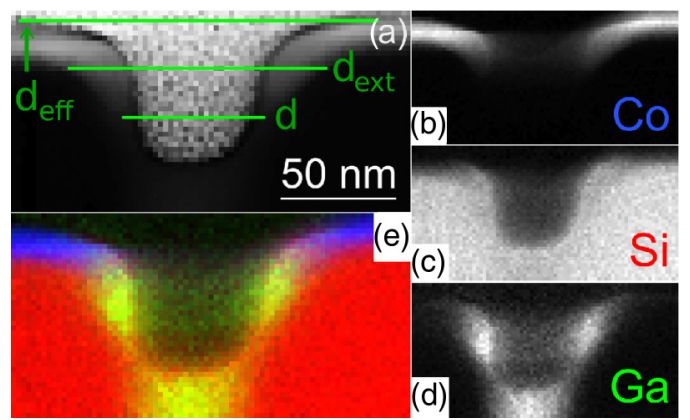

FIG. 4. (Color online) STEM-EDX chemical mapping of the array in Series 2-Co with $p=500 \mathrm{~nm}$ in the region around one of the holes, as hallmarked in Fig. 3(a). (a) High-angle annular dark-field (HAADF) imaging in which the diameters $d, d_{\mathrm{ext}}$, and $d_{\text {eff }}$ are indicated. Integrated X-rays intensity maps in (b) Co-K, (c) $\mathrm{Si}-\mathrm{K}$, and (d) Ga-K are composed with blue, red, and green colors in an RGB color map in (e)

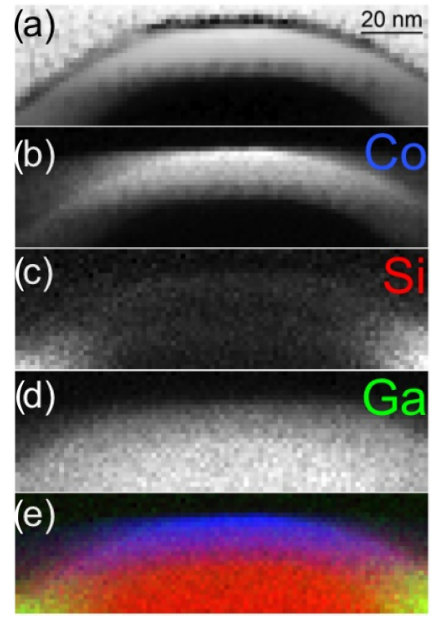

FIG. 5. (Color online) STEM-EDX chemical mapping of the array in Series 2-Co with $p=175 \mathrm{~nm}$ in the region between two holes, as hallmarked in Fig. 3(b). (a) HAADF image. Integrated x-rays intensity maps in (b) Co-K, (c) Si-K, and (c) Ga-K are composed with blue, red, and green colors in a RGB color map in (e).

chemical composition of the film is significantly modified. On the one hand, Ga implantation appears mainly in the antidot edges and bottom. On the other hand, Si intermixing or alloying with the cobalt of the film is evidenced by the apparent thickening of the cobalt film around the antidot edges. The relative compositions of the Ga-implanted $\mathrm{Co} / \mathrm{Si}$-intermixed area close to the hole averaged over an $8 \times 8 \mathrm{~nm}^{2}$ area are $\approx 15 \%$ at. $\mathrm{Co}, 15 \%$ at. $\mathrm{Ga}$, and $70 \%$ at. $\mathrm{Si}$.

In the case of $p=175 \mathrm{~nm}$, similar phenomena takes place with a $\mathrm{Ga}$ content up to $12 \%$ at. $\mathrm{Co} / \mathrm{Si}$ intermixing in this case gives rise to an interfacial Co-Si layer that stretches all along between the two antidots, with a relative composition of $33 \%$ at. $\mathrm{Co}$, and $58 \%$ at. $\mathrm{Si}$ in the area close to the hole. In the middle point between two holes, the presence of Ga is still negligible (below $2 \%$ at.), whereas the $\mathrm{Co} / \mathrm{Si}$ intermixing present a compositional ratio of $81 \%$ at. Si with respect to a $17 \%$ at. Co.

The increasing intermixing and decreased Ga concentration in the sample with $p=175 \mathrm{~nm}$ could be related to an increased dilution of the chemical species at the interface due to heating. This effect is expected to be stronger in the samples with smaller $p$, where proximity would cause the morphology and chemical composition of one hole to be modified during the etching of neighboring holes.

\section{MAGNETIC CHARACTERIZATION}

\section{A. Series 1: Study of antidot arrays on cobalt}

MOKE hysteresis loops for each array in Series 1 have been measured [32] with magnetic field in a range of $\pm 2500 \mathrm{Oe}$, large enough to saturate the magnetization, applied in-plane along the antidot rows. The coercivity, $H_{C}$ of the hysteresis loops of all the arrays are plotted in Fig. 6 as a function of $p$. The horizontal dashed line indicates the $H_{C}$ value of the unpatterned film, which is $14 \mathrm{Oe}$. The introduction of antidots in an array of $p=500 \mathrm{~nm}$ has already doubled $H_{C}$. The reduction in $p$, which implies an increase in the antidot density, 


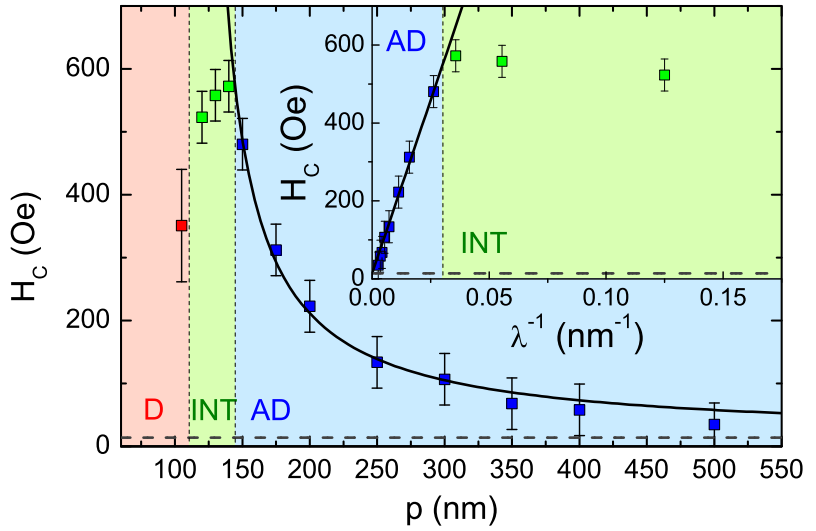

FIG. 6. (Color online) Series $1 . H_{C}$ vs $p$. Red, green, and blue colors represents $\mathrm{D}, \mathrm{INT}$, and $\mathrm{AD}$ regimes, respectively. The black line is the fit of $\Delta H_{C} \propto 1 / \lambda$, giving a fitting value for $d_{\text {eff }}=114 \pm 4 \mathrm{~nm}$. Dashed horizontal line indicates the coercivity of the unpatterned film, which is $14 \mathrm{Oe}$. In the inset, $H_{C}$ is plotted as a function of $1 / \lambda$.

leads to hysteresis loops with larger $H_{C}$. This is consistent with the picture of the antidots acting as pinning centers for magnetic domain walls. The maximum $H_{C}$ value obtained is 572 Oe, approximately 40 times larger than the unpatterned film coercivity, for $p=140 \mathrm{~nm}$. For $p>140 \mathrm{~nm}$, the expected linear trend with $\lambda^{-1}$ of the increase in $H_{C}$ with respect to that of the unpatterned film, $\Delta H_{C}=H_{C}-H_{C}^{\text {film }} \propto \lambda^{-1}$, is followed.

In Fig. 6 a black line represents the fit of $\Delta H_{C}$ to a function proportional to $1 / \lambda$. As the antidot edges are not sharp, there is a large uncertainty in the antidot diameter, so it is introduced as a fitting parameter, labeled as effective antidot diameter $d_{\text {eff }}$ (see Table I). The obtained value is $d_{\mathrm{eff}}=114 \pm 4 \mathrm{~nm}$, which is larger than the external diameter measured from SEM images $d_{\text {ext }}$, which is $107 \mathrm{~nm}$. This suggests that the FIB damaged ring of about 30-nm width surrounding the holes, previously characterized by TEM, is nonmagnetic. A probable cause is the local drop of saturation magnetization in this area caused by a local change in morphology and $\mathrm{Co} / \mathrm{Si}$ intermixing (see Fig. 4), that is a well known and previously reported effect [33]. From now on, $d_{\text {eff }}$ will be used for the definition of $\lambda$, i.e., $\lambda=p-d_{\text {eff }}$.

Surprisingly, a crossover in the dependence of $H_{C}$ on $p$ is found at $p=140 \mathrm{~nm}$, as it reaches a maximum and decreases when further decreasing $p$.

Considering the $\lambda$ value, Fig. 6 can be divided into three regions.

(1) Antidot (AD) regime: $p>140 \mathrm{~nm} \Rightarrow \lambda>26 \mathrm{~nm}$. The antidots are well separated from each other, and the expected tendency of $\Delta H_{C} \propto 1 / \lambda$ is followed (see Fig. 6, inset).

(2) Intermediate (INT) regime: $114 \mathrm{~nm}<p \leqslant 140 \mathrm{~nm} \Rightarrow$ $0<\lambda \leqslant 26 \mathrm{~nm}$. When, the distance between antidots $\lambda$ is small but still positive, a change in the $H_{C}$ dependence on geometry is found, as it smoothly decreases with $\lambda^{-1}$, in contrast to the linear increase in the AD regime. Therefore, although the magnetic material is still continuous, a different magnetic regime appears caused by the constrained space between antidots.

(3) Dot (D) regime: $p<114 \mathrm{~nm} \Rightarrow \lambda<0$. The cobalt film is still continuous, as $p>d$ for all the arrays, but $p<d_{\mathrm{eff}}$, and therefore the nonmagnetic regions intersect with each other. The magnetic material no longer a continuous film with holes, but an array of astroid-like dots (see Fig. 1) formed by the remaining magnetic material between the intersected antidots. This region does not appear in the inset of Fig. 6 as $\lambda^{-1}$ becomes negative.

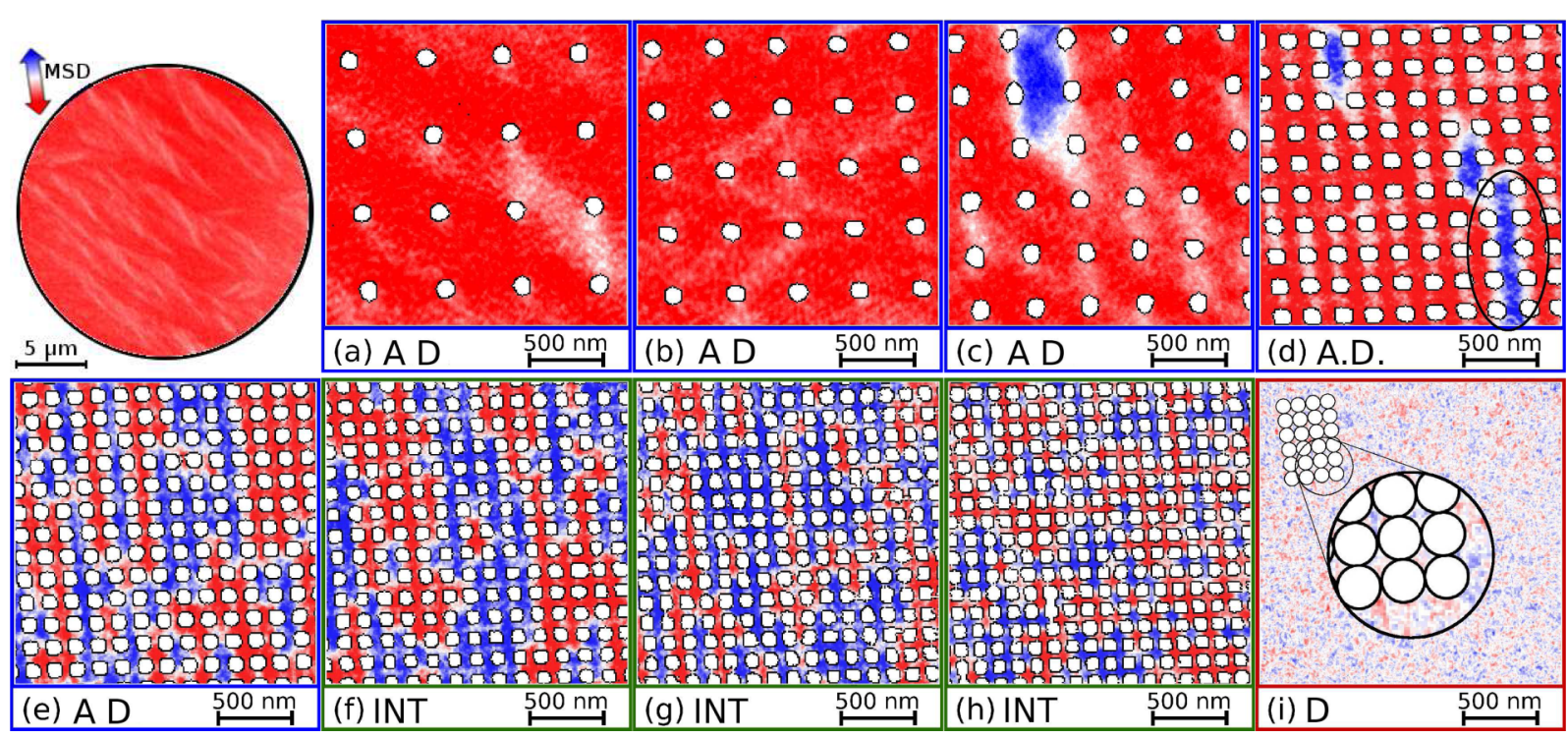

FIG. 7. (Color online) Series 1: XPEEM images of the unpatterned film, and the arrays of $p=$ (a) 500, (b) 400, (c) 300, (d) 200, (e) 150, (f) 140, (g) 130, (h) 120, and (i) $105 \mathrm{~nm}$. In a zoom the astroid-like shape of the dots formed by the intersection of the antidots can be apreciated. Blue and red colors in the images mean magnetization projection along the MSD pointing up and down, respectively. White color corresponds to magnetization either zero or perpendicular to the MSD. A superdomain is encircled in (d). A blue, green, or red frame surrounding each image indicates whether it belongs to the antidot (AD), intermediate (INT), or dot (D) regime. 
An alternative way of estimating the effective antidot diameter can be extracted from the difference in Kerr signal at positive and negative saturation $\Delta I_{r}$, as described by the authors of Ref. [34]. Details on the calculation can be found in the supplemental material [35]. The obtained effective diameter is $d_{\text {eff }}=112 \pm 5 \mathrm{~nm}$, which is in accordance with the result of the $\Delta H_{C} \propto 1 / \lambda$ fit (see Fig. 6), and again shows that the relevant antidot diameter is larger than the value observed with SEM.

To correlate the coercivity measurements with the magnetic domain structure of antidot arrays, XMCD images of the as grown state of a representative section of the arrays are shown in Fig. 7. The area covered by the antidots has been colored in white and the antidot edges in black. The detection of the antidots has been performed through image treatment with the ADVANCED WEKA SEGMENTATION plugin of the FIJI software [36]. Details in the process can be found in the supplemental material [37].

The image of the unpatterned film presents the magnetization ripple with small-angle domain walls typical of polycrystalline thin films. This evidences that the partial texturing during deposition has imposed an average magnetic easy axes, transverse to the ripple direction [9,38]. Due to the low coercivity of the film, parasitic magnetic fields applied during fabrication or measurement have been enough to saturate the sample, giving a negative mean projection of magnetization along the MSD.

In the $\mathrm{AD}$ regime [Figs. 7(a) to $7(\mathrm{e})]$ the magnetization configuration changes continuously as $p$ is reduced, from the typical magnetization ripple of the film in Fig. 7(a) $(p=500 \mathrm{~nm})$ to a structure completely dominated by the array geometry in Fig. 7(e) $(p=150 \mathrm{~nm})$. Regions with the same average magnetization, but with internal distribution to minimize the magnetic poles around the holes, are called superdomains [11]. They tend to assemble into a chain-like shape [12], as the one encircled in Fig. 7(d). Superdomain walls, i.e., boundaries between superdomains with different average magnetization orientation are, as expected, connecting adjacent antidots.

In the antidot arrays belonging to the INT regime [Figs. 7(f) to 7(h)], the superdomains' density increases when reducing $p$, while their shape changes from single column chains to a two-dimensional distribution, until the array of $p=120 \mathrm{~nm}$, in which no chain-like superdomain is observed. The size of the domains is decreased as well. All this together suggests a decrease in the correlation between unit cells of the array, i.e., a tendency to behave magnetically as an array of dots rather than an antidot array.

The array with the lowest period [ $p=105 \mathrm{~nm}$, Fig. 7(i)], which belongs to the $\mathrm{D}$ regime, has no magnetic contrast above the noise level, and therefore its XMCD image appears in white. Some antidots have been manually plotted in a small region for clarity.

A two-dimensional map of the magnetization angle $\theta$ can be obtained composing two images with different MSD. After a $45^{\circ}$ counterclockwise in-plane rotation of the sample, XMCD images of the arrays with $p=200$ (AD regime) and $120 \mathrm{~nm}$ (INT regime), have been measured to obtain the $\theta$ maps presented in Figs. 8 (AD) and 9 (INT), respectively. to quantify the magnetization direction, the arrows in the $\theta$ maps in Figs. 8

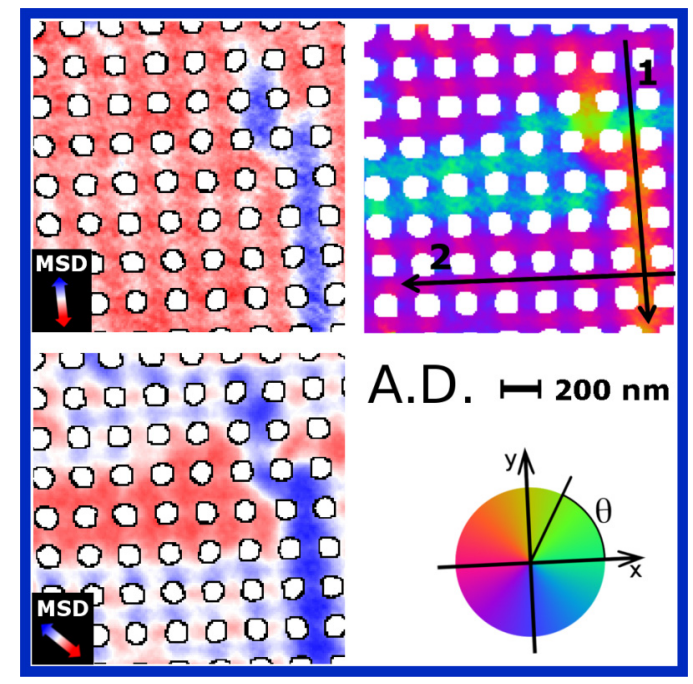

FIG. 8. (Color online) AD regime. Left: XMCD images of the array with $p=200 \mathrm{~nm}$, belonging to the $\mathrm{AD}$ regime, with MSD vertical (up), and $45^{\circ}$ tilted (down). Right: $\theta$ map of the magnetization angle.

and 9 represent the lines along which $\theta$ has been plotted as a function of position, $r$, in Figs. 10 and 11, respectively.

In the AD regime, (Fig. 8, $p=200 \mathrm{~nm}$ ), where chain-like domains were observed between two adjacent antidot columns in Fig. 7(d), the chains along antidot rows can also been observed in the $\theta$ map. The periodic $\theta(r)$ oscillations inside a superdomain observed in Fig. 10 correspond to that previously reported [11,12]. The magnetization direction tends to be diagonal in the wide area between four holes, but it aligns parallel to the antidot edges, that is, horizontally or vertically, when crossing the narrow necks between adjacent antidots.

In the INT regime, the $\theta$ map (Fig. 9, $\mathrm{p}=120 \mathrm{~nm}$ ) shows smaller domains, with a length of one or two unit cells of the

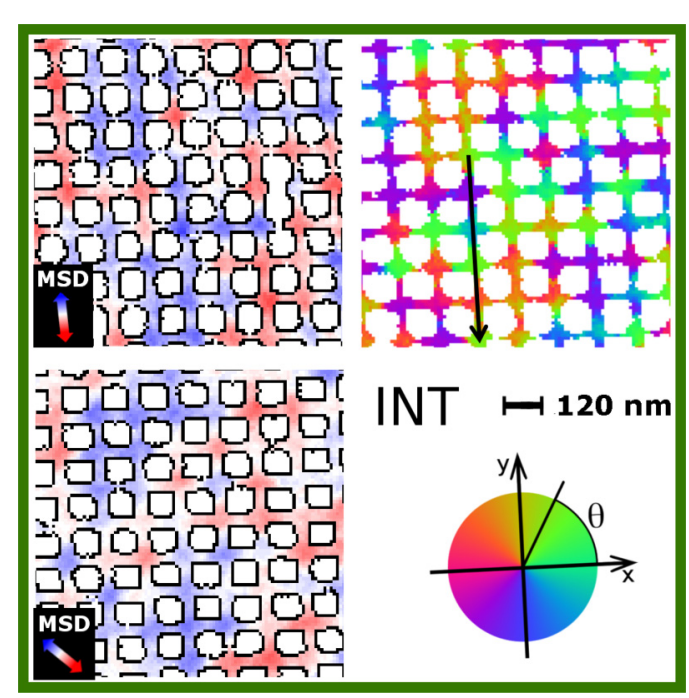

FIG. 9. (Color online) INT regime. Left: XMCD images of the array with $p=120 \mathrm{~nm}$, belonging to the INT regime, with MSD vertical (up), and $45^{\circ}$ tilted (down). Right: $\theta$ map of the magnetization angle. 


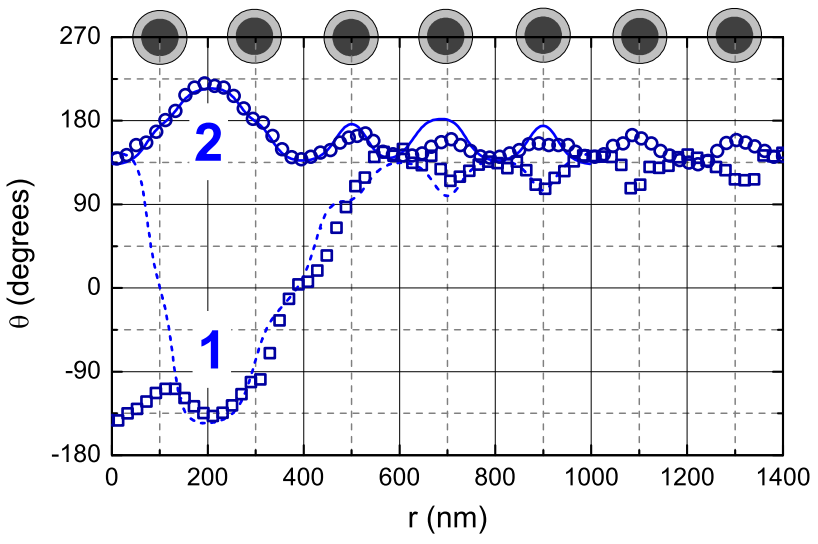

FIG. 10. (Color online) AD regime. $\theta(r)$ linescans in the array with $p=200 \mathrm{~nm}$, which belong to the AD regime. Experimental $\theta(r)$ along lines 1 and 2 indicated in Fig. 8 have been plotted as open squares and circles, respectively. Simulated $\theta(r)$ along lines 1 and 2 indicated in Fig. 15(a) are plotted as dashed and continuous lines, respectively. Circles on top of the graph represent the antidot positions. Holes with $d$ are plotted in dark grey and the FIB damaged ring with $d_{\text {eff }}$ in light grey.

array and abrupt transitions between them mainly in the necks between antidots. The $\theta(r)$ experimental linescan plotted in Fig. 11 shows no oscillations. Instead, abrupt transitions in the necks between holes separate domains of one or two unit cells of the array. Inside each domain the magnetization direction is approximately constant, pointing along one of the four diagonals.

The changes in the magnetic domain configuration between the $\mathrm{AD}$ and INT regimes are shown by the change in the $H_{C}$ trend with $p$. The magnetic correlation loss in the INT regime suggests that the magnetic behavior of the morphologicaly antidot array is closer to that of a dot array.

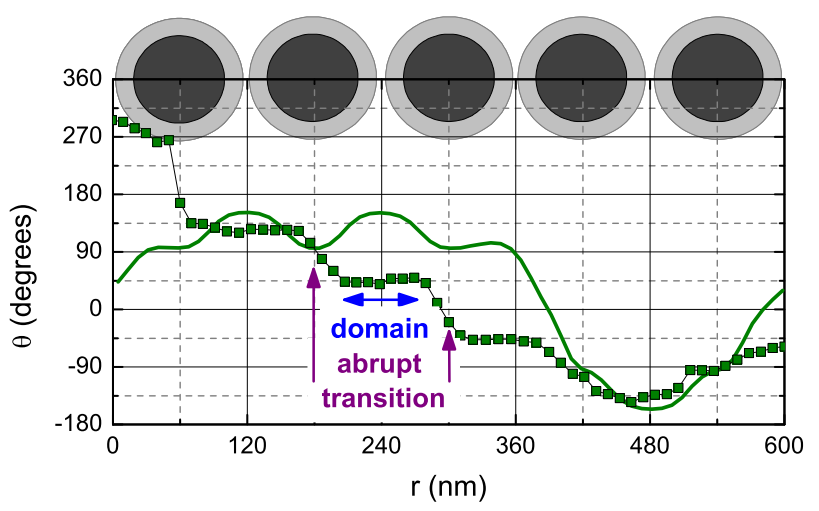

FIG. 11. (Color online) INT regime. $\theta(r)$ linescans in the array with $p=120 \mathrm{~nm}$, which belong to the INT regime. Experimental $\theta(r)$ along the line indicated in Fig. 9 has been plotted as open squares. The simulated linescan along the line indicated in Fig. 15(b) is plotted as a continuous line. Circles on top of the graph represent the antidot positions. Holes with $d$ are plotted in dark grey and the FIB damaged ring with $d_{\text {eff }}$ in light grey.

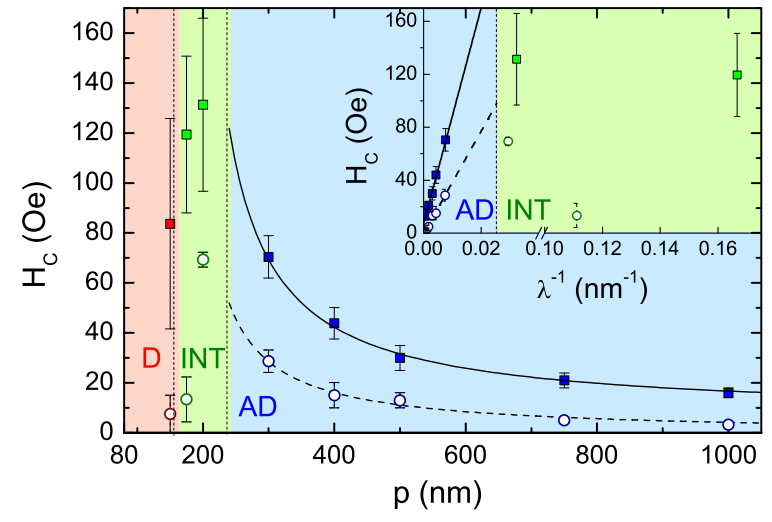

FIG. 12. (Color online) Series 2: $H_{C}$ dependence on $p$ of the antidot arrays on $\mathrm{Co}$ and $\mathrm{Py}$ are plotted as solid squares and open circles, respectively. The blue, green, and red colors represent data belonging to the $\mathrm{AD}$, INT, and $\mathrm{D}$ regimes, respectively. In the inset, $H_{C}$ vs $\lambda^{-1}$ is plotted, showing a linear trend for $p$ above the crossover. The function $\Delta H_{C} \propto 1 / \lambda$, with the obtained $d_{\text {eff }}$ value [35], is plotted as solid and dashed black lines for cobalt and permalloy, respectively.

\section{B. Series 2: Comparison between cobalt and permalloy antidot arrays}

MOKE hysteresis loops of the two series of antidot arrays on Co and Py have been measured in a range of magnetic fields of $\pm 2500 \mathrm{Oe}$, large enough to saturate their magnetization, applied in-plane along the direction of the antidot rows.

Following the same procedure as for Series 1, from the fit of $\Delta I_{r}$ an effective diameter of $d_{\mathrm{eff}}=169 \pm 4 \mathrm{~nm}$ and $166 \pm 4$ is obtained for Co and Py antidot arrays, respectively. Details can be found in the supplemental material [35].

The coercivity of the unpatterned film is $H_{C}^{\text {film }}=14$ and 2 Oe for Co and Py, respectively. The $H_{C}$ value of all the arrays is plotted as a function of $p$ in Fig. 12, both for Co and Py. For the largest $p=2 \mu \mathrm{m}, H_{C}$ is approximately that of the continous film for both materials. The maximum $H_{C}$ value is of 131 and 69.3 Oe for Co and Py, respectively, obtained for both at $p=200 \mathrm{~nm}$. This implies a maximum $H_{C}$ increase respect to the $H_{C}^{\text {film }}$ of nine times for Co, which is smaller than the 40 times increase in Series 1 . This is probably due to the larger dwell time of the ion beam in Series 2, causing larger FIB damage. In the case of Py, a maximum $H_{C}$ increase of 35 times is obtained.

The $H_{C}$ increase is, as expected, linear with $\lambda^{-1}$ for $p>200 \mathrm{~nm}$, corresponding to the AD regime. When further decreasing $p$ below that value, the same $H_{C}$ crossover to $\mathrm{D}$ regime, passing through the intermediate state, as in Series 1 is found. For both Co and Py films, a correlation of the three different regimes with the ones defined in Series 1 can be made just by rescaling the $p$ ranges, and the corresponding $\lambda$ ranges as follows:

(1) AD regime: $p>200 \mathrm{~nm} \Rightarrow \lambda>31 \mathrm{~nm}$;

(2) INT regime: $169 \mathrm{~nm}<p<200 \mathrm{~nm} \Rightarrow 0<\lambda \leqslant$ $31 \mathrm{~nm}$;

(3) D regime: $p<169 \mathrm{~nm} \Rightarrow \lambda<0$. 
This result proves that the described phenomenon occurs not only in cobalt, but is extensive to Py, i.e., to other materials. The limits between different regimes appear at the same $\lambda$ values for all the studied arrays, fabricated on two different materials, Co and Py, and with two different sets of geometrical parameters. Therefore, it can be concluded that the regime threshold parameters depend only on the exchange length of the material $\Delta_{d}$.

\section{MICROMAGNETIC SIMULATIONS}

Micromagnetic simulations of the magnetization reversal process of a cobalt thin film containing antidot arrays were performed using the OOMMF [39] program. The modeled system consists of $5 \times 5$ antidots arranged into a square array, and two-dimensional (2D) periodic boundary conditions. The diameter of antidots ranged in the interval $80 \leqslant d \leqslant 120 \mathrm{~nm}$ and the array period $70<p<250 \mathrm{~nm}$. The experimental D regime, where $p<d_{\text {eff }}$, is simulated by including the cases when $p<d$. The discretization size was equal to $2 \mathrm{~nm}$ for the smallest system and $4 \mathrm{~nm}$ for the largest one, in any case below $\Delta_{d}$. The following micromagnetic parameters were used: the exchange constant $\mathrm{A}=30 \times 10^{-12} \mathrm{~J} / \mathrm{m}$, and the saturation magnetization $\mathrm{M}_{\mathrm{S}}=1400 \times 10^{3} \mathrm{~A} / \mathrm{m}$. Since because of the polycrystalline structure of the film the magnetocrystalline anisotropy is negligibly small, we set this parameter to zero in the simulations.

The hysteresis loops were obtained by applying the magnetic field in-plane, parallel to the antidot rows. A selection of them can be found in the supplemental material [32].

The dependence of the hysteresis loop coercivity, $H_{C}$, on $\lambda$ for selected $d$ values is presented in Fig. 13. The curves for diferent diameters show a similar behavior, presenting a maximum $H_{C}$ value, $H_{C}^{\max }$, at critical geometrical dimensions when $p$ is slightly larger than $d$, that is, $\lambda \gtrsim 0$.

$H_{C}^{\max }$, and the $p$ at which it appears $p_{\max }$, are plotted as a function of $d$ in Fig. 14. $H_{C}^{\max }$ decreases for increasing $d$, which is consistent with the experimental results. The coercivity

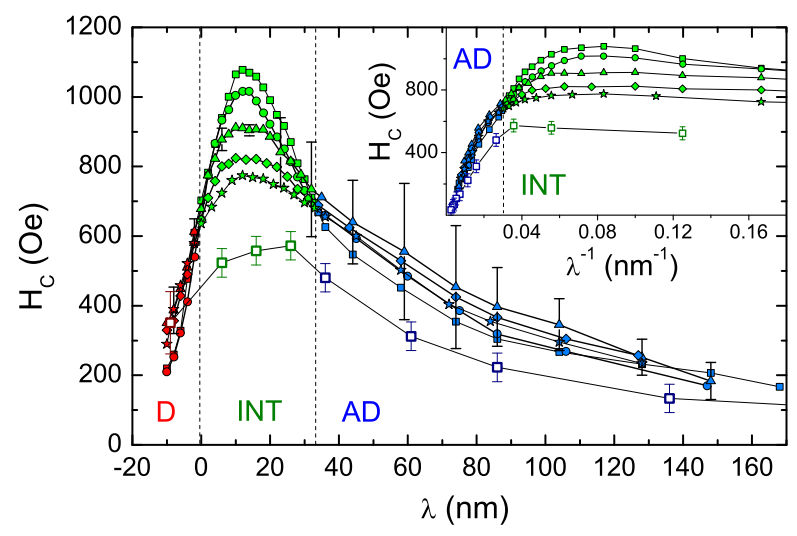

FIG. 13. (Color online) $H_{C}$ of simulated arrays of antidots with $d=80 \mathrm{~nm}$ (squares), $90 \mathrm{~nm}$ (circles), $100 \mathrm{~nm}$ (triangles), $110 \mathrm{~nm}$ (rhombs), and $120 \mathrm{~nm}$ (stars), as a function of $\lambda$. Experimental values for Series 1 are plotted as open squares for comparison. The data belonging to the antidot, intermediate, and $\mathrm{D}$ regimes are represented with blue, green, and red colors, respectively. In the inset the same data set is plotted vs $\lambda^{-1}$.

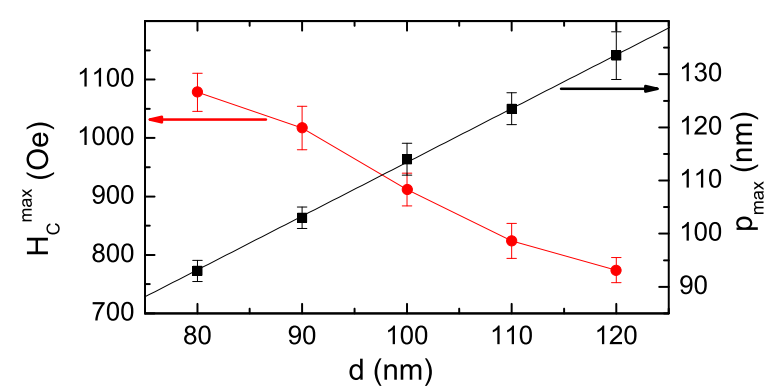

FIG. 14. (Color online) Values of $H_{C}^{\max }$ and $p_{\max }$ as a function of $d$ for $H_{C}$ curves of different diameters. The linear fit of $p_{\max }$ vs $d$ confirms that the $H_{C}^{\max }$ allways occurs for $p=d+12.3 \mathrm{~nm}$.

maximum occurs at $p_{\max }=d+12.3 \mathrm{~nm}$, that is, at a constant $\lambda=12.3 \mathrm{~nm}$ for all the calculated diameters.

The $\lambda$ value at the lower limit of the AD regime coincided in the experiments with $p_{\max }$. However, it can be assessed from the inset of Fig. 13 that in simulations, the lower limit of the $\mathrm{AD}$ regime appears at smaller $\lambda^{-1}$ values than the position of the $H_{C}^{\max }$ or, what is the same, larger $\lambda$ values. As the limit between the antidot and INT regimes is thus not well defined, it has been set to $\lambda=28 \mathrm{~nm}$ in analogy to the experimental value. Thereby, the three regimes can be described from the simulations results as following.

(1) AD regime: $\lambda>28 \mathrm{~nm}$. There is nucleation, pinning, and therefore jumps in $M(H)$. As $\lambda$ decreases, there is less mobility of DWs, and $H_{C}$ increases.

(2) INT regime: $0<\lambda \leqslant 28 \mathrm{~nm}$. As $\lambda$ is reduced, DW propagation becomes harder, and the linear dependence of $\Delta H_{C}$ on $\lambda^{-1}$ is gradually lost. As $\lambda$ is further decreased, the necks become narrower, and the area that has to be nucleated with a reversed domain is smaller. As a consequence, $H_{C}$ decreases.

(3) D regime: $\lambda<0$. The area between antidots, i.e., the "dot" magnetic material left in between, has an astroid-like shape [see Fig. 15(c)]. When $\lambda=0$, the astroid-like cusps are sharp, forming a zero angle. When $\lambda$ is further decreased this angle is less acute and the shape becomes closer to a square dot. Simulations show that the nucleation of the domain starts at one of the cusps, and the moments rotate towards the opposite direction in continuous, and a nonhomogeneous way (noncoherent moment reversal). As the astroid cusp angle is less-acute (that is, as $\lambda$ decreases), it is easier for the reversed domains to be nucleated. This implies that $H_{C}$ decreases when decreasing $\lambda$.

In Fig. 13 the experimental values of Series 1 are plotted as open squares. Simulated $H_{C}$ is larger than the experimental one, as expected due to the absence of random defects that facilitate nucleation of reversed domains. The curves that better fit the experimental values are the ones for diameter 110 and $120 \mathrm{~nm}$, as they are closer to the experimental $d_{\text {eff }}$. Experimentally, in dot and INT regime $H_{C}$ decreases more smoothly when decreasing $\lambda$ than in simulations. This is probably due to the bluntness of the experimental antidot edges.

As previously done with experimental data, images of the $\theta$ map of the magnetization are presented in Fig. 15 for simulations of arrays with $d=110 \mathrm{~nm}$ and $p=200 \mathrm{~nm}$ 

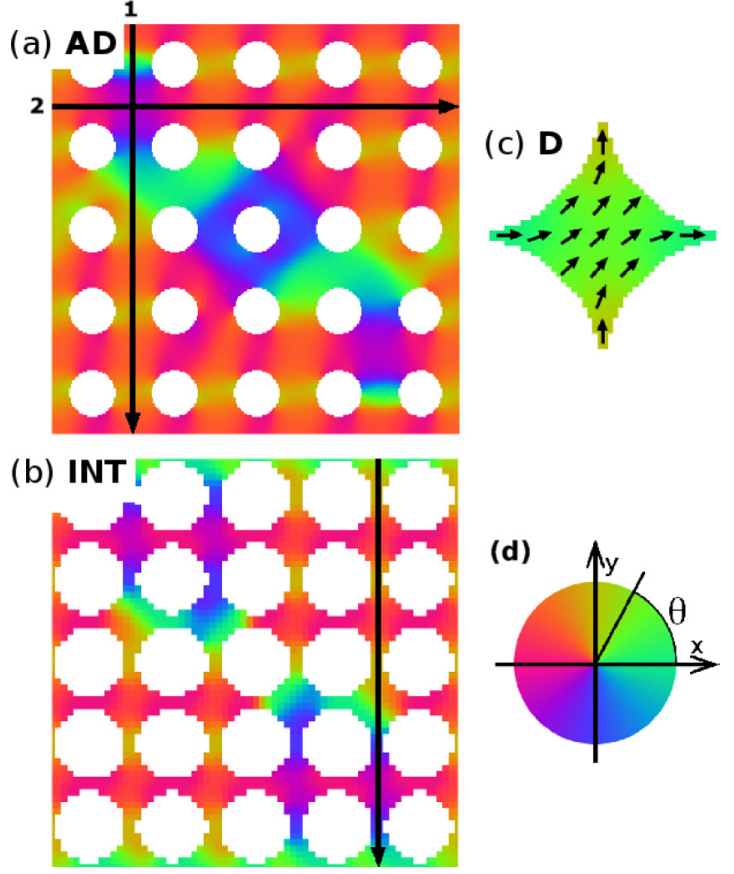

FIG. 15. (Color online) Simulated $\theta$ maps of the magnetization angle of antidot arrays with $d=110 \mathrm{~nm}$ belonging to the (a) AD and (b) INT regimes, with $p=200$ and $120 \mathrm{~nm}$, respectively. (c) $\theta$ map of the remanent state of a dot of the $\mathrm{D}$ regime array with $p=d=110 \mathrm{~nm}$. (d) Colorscale of $\theta$.

[Fig. 15(a)], $120 \mathrm{~nm}$ [Fig. 15(b)], and $110 \mathrm{~nm}$ [Fig. 15(c)], being, respectively, in the antidot, intermediate, and $\mathrm{D}$ regimes. In the array of $p=200 \mathrm{~nm}$, the magnetization is distributed in well-defined superdomains, as observed in the equivalent experimental image, in Fig. 8. The simulated $\theta(r)$ linescans along the lines labeled in Fig. 15(a) as 1 and 2 are plotted together with the experimental ones in Fig. 10. They clearly show the typical oscillations of $\theta(r)$ present in superdomains, and there is a good agreement with experimental $\theta(r)$ linescans.

The array of $p=120 \mathrm{~nm}$ shows smaller domains with very strong DW pinning. The corresponding $\theta(r)$ linescan from simulations, plotted in Fig. 11, still shows the $\theta(r)$ oscillations typical from superdomains. Thus, the simulated $\theta(r)$ in the INT regime resembles that of the $\mathrm{AD}$ regime, in contrast to the experimental $\theta(r)$, which is close to that expected in the $\mathrm{D}$ regime.

Figure 15(c) shows an astroid-like dot formed by the remaining magnetic material left between the intersected antidots. Due to the astroid-like shape of the dots, the magnetization at remanence is distributed in a nonhomogeneous state, which we have named the astroid state.

\section{DISCUSSION AND CONCLUSION}

The previous results suggest an unified description of the magnetic properties of patterned magnetic thin films along the crossover from antidot (AD) to dot (D) regime that takes place through an unexpected intermediate (INT) regime.

The combination of challenging fabrication and experimental techniques such as FIB and XPEEM allowed the necessary access to the nanometric scale, while micromagnetic simulations led to successful hallmark of the experimental results. The antidot arrays were fabricated by means of FIB etching. This resulted, as proven by HRTEM experiments, in a ring surrounding the antidots with implantated gallium ions and $\mathrm{Co} / \mathrm{Si}$ intermixing. The damaged area caused a broadening of the effective antidot diameter, enabling the case where $p<d_{\text {eff }}$ and antidots intersect with each other forming an array of isolated dots. The crossover between the $\mathrm{AD}$ and $\mathrm{D}$ regimes has been studied for two $d_{\mathrm{eff}}$ values, and two different materials (Co and Py), with the focus on the INT regime.

In the $\mathrm{AD}$ regime $\left(\lambda \gg \Delta_{d}\right)$ the interantidot spacing is large enough to sustain inhomogeneous magnetization structures, such as DWs. DWs can propagate through the antidot array, although the pinning effect of the antidots is stronger the closer they are to each other. Therefore, $H_{C}$ increases when $\lambda$ is reduced, following the empirical law according to which $\Delta H_{C} \propto 1 / \lambda$. In the diluted regime $(\lambda \gg \mathrm{DW}$ width) the domain configuration resembles the ripple pattern in the unpatterned film. When $\lambda$ is decreased the geometry dominates, favoring the nucleation of superdomains in chains along antidot columns and rows, with a very good agreement with previous observations [11,12].

In the $\mathrm{D}$ regime $(\lambda<0)$, the intersecting antidots divide the film into astroid-like shaped dots. A nonhomogeneous magnetization distribution along each dot has been predicted by means of micromagnetic simulations, and the magnetic state has been named the astroid state. The magnetization switching occurs in each dot via noncoherent moment rotation, starting in one cusp and propagating to the whole dot. This results in $H_{C}$ decreasing when $\lambda$ is reduced, contrary to its trend in the $\mathrm{AD}$ regime.

A fascinating INT regime appears in the crossover from $\mathrm{AD}$ to $\mathrm{D}$ regime, at the same $\lambda \approx 30 \mathrm{~nm}$ in all the studied arrays. This threshold parameter is independent of the hole diameter and the material. This proves that the phenomenom is not only extensive to other materials, but it only depends on the exchange length of the material $\Delta_{d}$, which is the same for both Co and Py. Thus, it can be concluded that the phenomena follows a corresponding state behavior. In the INT regime, $H_{C}$ increases with $\lambda$, as in the $\mathrm{D}$ regime. XMCD images show that the domain structure changes from chain-like superdomains at large $\lambda$ arrays, to smaller domains, with constant magnetization and abrupt transitions between them for small $\lambda$ arrays. This reveals a loss of correlation between the unit cells of the array. All these results evidence that the magnetic behavior of the morphologically antidot arrays in this regime is closer to dot arrays than to antidot arrays.

Micromagnetic simulations of arrays on cobalt thin films support the obtained results, showing a similar $H_{C}$ trend with $\lambda$. The threshold $\lambda$ value is also independent from the antidot diameter in simulations, strengthening the evidence of a corresponding state behavior.

In conclusion, the study of the INT state has revealed a dual behavior, in which a morphologically antidot array behaves magnetically as an array of isolated dots. This work points towards a new strategy in magnetic nanotechnology for fabricatig arrays of magnetic bits, i.e., basic elements for magnetic information storage (magnetic dots), embedded into a continuous 2D structural system. 


\section{ACKNOWLEDGMENTS}

The financial support of MINECO-FEDER MAT2011/ 23791, MAT2012-38318-C03-03, MAT2012-36844, FIS2010-20979-C02-02, and Basque Government Project No. PI2012-47 is acknowledged. C. Castán-Guerrero and
K. J. Merazzo acknowledge Ph.D. grants from CSIC and the University of Costa Rica. The work of P. Gawronski was partially supported by the Polish Ministry of Science and Higher Education and its grants for Scientific Research and by PL-Grid Infrastructure. We acknowledge J. Bryson for his assistance with the FIJI software.
[1] B. D. Terris and T. Thomson, J. Phys. D: Appl. Phys. 38, R199 (2005).

[2] J. M. Daughton, A. V. Pohm, R. T. Fayfield, and C. H. Smith, J. Phys. D 32, R169 (1999).

[3] V. V. Kruglyak, S. O. Demokritov, and D. Grundler, J. Phys. D 43, 264001 (2010).

[4] S. Neusser and D. Grundler, Adv. Mater. 21, 2927 (2009).

[5] H. Masuda and K. Fukuda, Science 268, 1466 (1995).

[6] X. Zhang, A. V. Whitney, J. Zhao, E. M. Hicks, and R. P. Van Duyne, J. Nanosci. Nanotechnol. 6, 1920 (2006).

[7] J. Baglin, Appl. Surf. Sci. 258, 4103 (2012).

[8] V. R. Manfrinato, L. Zhang, D. Su, H. Duan, R. G. Hobbs, E. A. Stach, and K. K. Berggren, Nano Lett. 13, 1555 (2013).

[9] A. Hubert and R. Schäfer, Magnetic Domains (Springer, New York, 1998).

[10] K. Theis-Bröhl, A. Westphalen, H. Zabel, U. Rücker, J. McCord, V. Höink, J. Schmalhorst, G. Reiss, T. Weis, D. Engel, A. Ehresmann, and B. P. Toperverg, New J. Phys. 10, 093021 (2008).

[11] X. K. Hu, S. Sievers, A. Müller, V. Janke, and H. W. Schumacher, Phys. Rev. B 84, 024404 (2011).

[12] L. J. Heyderman, F. Nolting, D. Backes, S. Czekaj, L. LopezDiaz, M. Kläui, U. Rüdiger, C. A. F. Vaz, J. A. C. Bland, R. J. Matelon, U. G. Volkmann, and P. Fischer, Phys. Rev. B 73, 214429 (2006).

[13] R. P. Cowburn, A. O. Adeyeye, and J. A. C. Bland, Appl. Phys. Lett. 70, 2309 (1997).

[14] R. Cowburn, A. Adeyeye, and J. Bland, J. Magn. Magn. Mater. 173, 193 (1997).

[15] P. Vavassori, G. Gubbiotti, G. Zangari, C. T. Yu, H. Yin, H. Jiang, and G. J. Mankey, J. Appl. Phys. 91, 7992 (2002).

[16] L. J. Heyderman, F. Nolting, and C. Quitmann, Appl. Phys. Lett. 83, 1797 (2003).

[17] E. Mengotti, L. J. Heyderman, F. Nolting, B. R. Craig, J. N. Chapman, L. Lopez-Diaz, R. J. Matelon, U. G. Volkmann, M. Klaui, U. Rudiger, C. A. F. Vaz, and J. A. C. Bland, J. Appl. Phys. 103, 07D509 (2008).

[18] P. Gawroński, K. J. Merazzo, O. Chubykalo-Fesenko, A. Asenjo, R. P. D. Real, and M. Vázquez, Europhys. Lett. 100, 17007 (2012).

[19] I. Ruiz-Feal, L. Lopez-Diaz, A. Hirohata, J. Rothman, C. Guertler, J. Bland, L. Garcia, J. Torres, J. Bartolome, F. Bartolome, M. Natali, D. Decanini, and Y. Chen, J. Magn. Magn. Mater. 242-245, 597 (2002).

[20] E. Paz, F. Cebollada, F. J. Palomares, J. M. González, M.-Y. Im, and P. Fischer, J. Appl. Phys. 111, 073908 (2012).
[21] K. Pirota, P. Prieto, A. Neto, J. Sanz, M. Knobel, and M. Vazquez, J. Magn. Magn. Mater. 320, e235 (2008).

[22] P. Prieto, K. R. Pirota, M. Vazquez, and J. M. Sanz, Phys. Status Solidi A 205, 363 (2008).

[23] M. Vázquez, K. Pirota, D. Navas, A. Asenjo, M. HernándezVélez, P. Prieto, and J. Sanz, J. Magn. Magn. Mater. 320, 1978 (2008).

[24] A. Lebib, S. P. Li, M. Natali, and Y. Chen, J. Appl. Phys. 89, 3892 (2001).

[25] S. Goolaup, A. O. Adeyeye, and N. Singh, Phys. Rev. B 73, 104444 (2006).

[26] C. A. Ross, S. Haratani, F. J. Castaño, Y. Hao, M. Hwang, M. Shima, J. Y. Cheng, B. Vögeli, M. Farhoud, M. Walsh, and H. I. Smith, J. Appl. Phys. 91, 6848 (2002).

[27] W. Scholz, K. Guslienko, V. Novosad, D. Suess, T. Schrefl, R. Chantrell, and J. Fidler, J. Magn. Magn. Mater. 266, 155 (2003).

[28] R. P. Cowburn, J. Phys. D: Appl. Phys. 33, R1 (2000).

[29] C. Castán-Guerrero, J. Sesé, J. Bartolomé, F. Bartolomé, J. Herrero-Albillos, F. Kronast, P. Strichovanec, K. J. Merazzo, M. Vázquez, P. Vavassori, and L. M. García, J. Nanosci. Nanotechnol. 12, 7437 (2012).

[30] E. Nikulina, O. Idigoras, P. Vavassori, A. Chuvilin, and A. Berger, Appl. Phys. Lett. 100, 142401 (2012).

[31] F. Kronast, J. Schlichting, F. Radu, S. Mishra, T. Noll, and H. Dürr, Surf. Interface Anal. 42, 1532 (2010).

[32] See Supplemental Material at http://link.aps.org/supplemental/ 10.1103/PhysRevB.89.144405 for a selection of the hysteresis loops of some of the arrays in Series 1.

[33] J. Fassbender and J. McCord, J. Magn. Magn. Mater. 320, 579 (2008).

[34] C. Castán-Guerrero, J. Bartolomé, F. Bartolomé, L. M. García, J. Sesé, P. Strichovanec, J. Herrero-Albillos, K. J. Merazzo, M. Vázquez, and P. Vavassori, J. Korean Phys. Soc. 62, 1521 (2013).

[35] See Supplemental Material at http://link.aps.org/supplemental/ 10.1103/PhysRevB.89.144405 for details of the calculation of $d_{\text {eff }}$ from the Kerr signal at saturation.

[36] J. Schindelin, I. Arganda-Carreras, E. Frise, V. Kaynig, M. Longair, T. Pietzsch, S. Preibisch, C. Rueden, S. Saalfeld, B. Schmid, J.-Y. Tinevez, D. J. White, V. Hartenstein, K. Eliceiri, P. Tomancak, and A. Cardona, Nat. Methods 9, 676 (2012).

[37] See Supplemental Material at http://link.aps.org/supplemental/ 10.1103/PhysRevB.89.144405 for details in the detection of holes in the XMCD images.

[38] E. Feldtkeller, J. Appl. Phys. 34, 2646 (1963).

[39] M. Donahue and D. Porter, OOMMF computer code, http://math.nist.gov/oommf 